\title{
Design of a New Nonlinear Stiffness Compliant Actuator and Its Error Compensation Method
}

\author{
Shaobin Lan and Zhibin Song \\ Key Laboratory of Mechanism Theory and Equipment Design of Ministry of Education, Tianjin University, Tianjin 300072, China \\ Correspondence should be addressed to Zhibin Song; songzhibin@tju.edu.cn
}

Received 17 June 2016; Revised 10 September 2016; Accepted 4 October 2016

Academic Editor: Huosheng $\mathrm{Hu}$

Copyright (C) 2016 S. Lan and Z. Song. This is an open access article distributed under the Creative Commons Attribution License, which permits unrestricted use, distribution, and reproduction in any medium, provided the original work is properly cited.

\begin{abstract}
Compliant actuators are more advantageous than stiff actuators in some circumstances, for example, unstructured environment robots and rehabilitation robots. Compliant actuators are more adaptive and safe. Constant stiffness compliant actuators have some limitations in impedance and bandwidth. Variable stiffness actuators improve their performance owing to introducing an extra motor to tune the stiffness of the actuators. However, they also have some limitations such as the bulky structure and heavy weight. It was also found that there are some waste functions existing in the current variable stiffness actuators and that the fully decoupled position control and stiffness tune are not necessary, because there exist some regular phenomena during most circumstances of human interaction with the robots which are "low load, low stiffness and high load, high stiffness". In this paper, a design method for nonlinear stiffness compliant actuator was proposed which performed the predefined deflection-torque trajectory of the regular phenomenon. A roller and a cantilever which has special curve profile constitute the basic mechanical structure of the nonlinear stiffness compliant actuators. An error compensation method was also proposed to analyze the stiffness of elastic structure. The simulation results proved that the proposed method was effective in designing a predefined nonlinear stiffness compliant actuator.
\end{abstract}

\section{Introduction}

Traditional actuators used in robotics or machines are stiff actuators which could obtain good position control and bandwidth. However, since gear boxes are usually used in stiff actuators, the backdrivability is always poor, which is not suitable in some circumstances, for example, humanmachine interaction, bioinspired robots and unstructured environment robots, and rehabilitation robots. The compliant actuator using an elastic component between the actuator and load was first proposed by Pratt in MIT [1], which is also called series elastic actuator (SEA). This kind of passive compliant actuator can be looked at as a pure force source, which is able to perform perfect force control.

From the early SEA with a linear spring to the later one with a torsional spring [1-3], then to the constant stiffness SEA optimized via finite element methods [4], and finally to the latest SEA with variable stiffness [5], passive compliant actuators were greatly improved owing to more compact structure and wider bandwidth of force control $[6,7]$. The variable stiffness SEA has attracted lots of interests and developed very quickly recently. Summarizing these proposed ideas, they could be divided into three groups to obtain the property of variable stiffness [8]:

(1) Change transmission between the load and elastic structures. AwAS [9] implemented the adjustable stiffness of its actuator via changing the effective length of linkages. AwAS-II [10], CompAct-VSA [11], and VSTA [12] varied their stiffness of actuators through adjusting the fulcrum position.

(2) Change the preload of the spring. FSJ [13], BAVS [14], and BAFSA [15] changed the stiffness of actuators via changing the contact position between the roller and cam mechanism with compressing the springs.

(3) Change the physical structure of springs. Jack Spring [16] and others obtained the variable stiffness actuator through changing the number of effective spring coins.

In general, most of variable stiffness actuators use two actuator units: one for position control and the other for the stiffness regulation, which makes the position control and stiffness regulation independent. Of course, variable stiffness 
actuator is flexible to adjust the stiffness whatever the position of rotor is. However, it also leads to the bulky and complicated mechanical structure [17]. On the other hand, decoupled position control and stiffness regulation in most of variable stiffness actuators are not used sufficiently.

Few researchers studied nonlinear stiffness SEA without an additional motor. Migliore et al. developed a nonlinear stiffness SEA with antagonistic nonlinear elastic springs [18]. Palli et al. also designed a kind of nonlinear stiffness SEA [19]. The nonlinear stiffness mimics that of the biological joints [20]. It was noted there was a common property among these researches that the stiffness of actuators increases with the load increasing. It could be summarized as "low load, low stiffness and high load, high stiffness," which is also common in human activity. Human limbs will stiffen to lift a heavy object but soften to clean up with a whisk.

This functionality is useful for human to obtain dexterous performance with low load and stability with high load [21]. To obtain the better running performance, researchers in MIT Biomimetic Lab developed MIT Cheetah robot with quadratic elastic stiffness in robot's ankle joint [22]. Omer et al. proposed an adjustable stiffness joint for a bipedal robot where two linear springs with different stiffness were adopted and assembled in parallel with an offset. When lower load was given to the output link, the spring with lower stiffness was compressed and the stiffness of system is low. When the spring of low stiffness had been compressed fully, the spring with high stiffness was compressed and the stiffness of system became high [23]. Torrealba and Udelman proposed an adjustable compliant actuator by designing a particular cam shape. The stiffness can be adjusted by compressing the floating spring, and a group of torque-deflection curves show that the stiffness increased with respect to the deflection [15]. Kilic et al. proposed a wrapping cam mechanism with a torsion spring to attain quadratic behavior [24]. Palli et al. utilized a compliant four linkages' mechanism as topology structure and optimized its dimensions of each bar using pseudo-rigid-body model to obtain the nonlinear relationship between position and stiffness of the actuator [19]. The different methods were used to generate nonlinear stiffness of compliant actuator. However, the feasible theoretical method to design the compliant mechanism which possesses the predefined nonlinear stiffness has not been given yet.

In this paper, a new design method of compliant actuators with predefined nonlinear stiffness trajectory of "low load, low stiffness and high load, high stiffness" was proposed. The basic structure of the proposed mechanism consisted of a roller, an elastic component, and a contact part. One joint of 3-DOF (degrees of freedom) rehabilitation robot for shoulder training was introduced as the compliant actuator with defined nonlinear stiffness. Theory deduction and resolution process using software were described in detail. The simulation and experiments were also conducted.

\section{Mechanical Design of the Compliant Actuator}

In this part, one compliant joint of a rehabilitation robot for shoulder complex was introduced which used the proposed
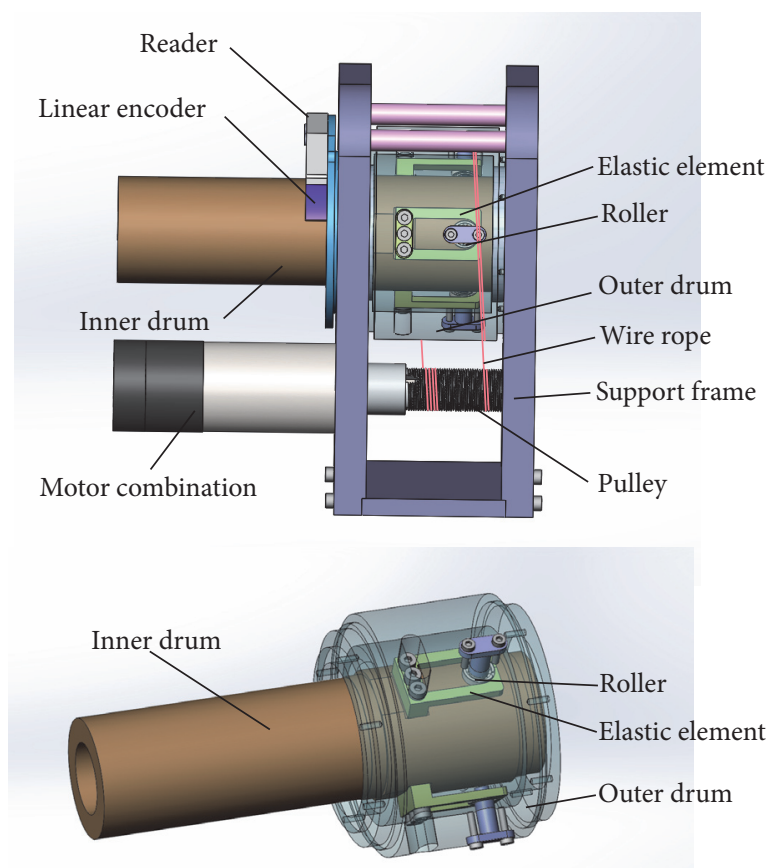

Figure 1: CAD model of the first joint of rehabilitation robot.

nonlinear elastic elements. This robot was designed with $3 \mathrm{DOF}$ (RRP configuration) by International Center of Advanced Mechanisms and Robotics (iCAMAR) of Tianjin University. All of these joints installed the nonlinear elastic elements and the mechanical design of the first joint was introduced as an example which is shown in Figure 1. The compliant joint consists of an inner drum, an outer drum, elastic elements, rollers, a linear magnetic encoder, wire rope, a pulley, motor combination (including a motor, gear box, and an encoder), and support frame. The inner drum connects with external loads and the outer drum is driven by a BLDC motor (Maxon) via wire rope and the pulley. To implement bidirectional compliant actuation, each compliant mechanism consists of two symmetrically arranged elastic elements and a roller. There are three compliant mechanisms distributed on the surface of inner drum with 120 degrees among them. The elastic elements connect with the inner drum and the rollers are installed on the outer drum via flanges. Bearings are used as rollers in order to decrease friction. A linear encoder and a reader which constitute linear magnetic encoder are installed on the outer drum and inner drum, respectively, so that the relative motion between them can be detected precisely. The equilibrium status is that the roller is located in the center line of two symmetrical elastic elements.

The key issue of the design of a nonlinear stiffness compliant actuator is to design elastic mechanisms with nonlinear stiffness. In this paper, a very basic and common elastic structure was adopted as the elastic element, namely, cantilever structure, which is easy to design and accurate to calculate. The main idea of topology design is to propose an elastic element composed of the elastic part and the contact 


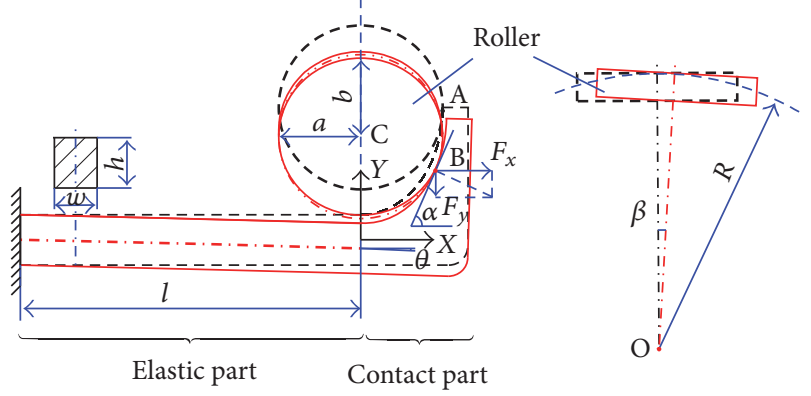

FIGURE 2: The schematic diagram of force analysis of the proposed mechanism.

part which has a special curve profile contacting with a roller. The impedance in the direction of roller motion could be changed when the slope of the tangent plane between the roller and the contact part varied, and then the mechanical stiffness of system changed.

\section{Methodology of the Curve Profile Design}

The core of designing the nonlinear stiffness compliant actuator is the nonlinear elastic mechanism. As we investigated from the current literature, there is no method to obtain the mechanism whose stiffness matches the predefined nonlinear stiffness trajectory. In this paper, we proposed a simple mechanism to achieve it, which consists of an elastic element and a roller. The elastic element consists of two parts: the elastic part and the contact part. The elastic part is a uniform cantilever which will generate deflection and rotation when it is given a certain external force. The contact part has a specific curve profile.

Figure 2 shows the schematic diagram of the proposed basic nonlinear stiffness mechanism. According to this figure, the elastic element was compressed by the roller when force was exerted on the curve profile of the contact part. The stiffness would be zero when the roller moved on the tangent plane at the contact point and generated contact force was zero. Alternatively, the stiffness would be maximum when the roller moved perpendicularly to the tangent plane at the contact point. Between both of extremity situations, due to the deflection and deflection angle of the cantilever, the angle between the direction of roller motion and the tangent plane would be within 0-90 degrees. The relationship between the roller motion and components of contact force along the roller motion could be adjusted with the slope of contact point, so that the stiffness could be changed.

The force analysis in the mechanism is also shown in Figure 2 where a roller compresses the elastic element. Solid red line shows the current status of the roller and the elastic element and dotted black line shows the last status. Point A represents the contact position between the roller and the elastic element in past status. Point B shows the current contact position of them. $\mathrm{C}$ is the center of ellipse in vertical view when the roller rotates around the point O. From the axial direction of the outer drum, the roller which is installed on a rotational component moves along the arc of radius $R$.
The rest of the symbols and their description during force and motion analysis of the proposed mechanism are shown in the Abbreviations.

To simplify analysis of the deflection of the elastic element, the elastic element was analyzed by divided it into two parts. One is a simple cantilever and the other is variable cross section cantilever. Generally speaking, the deflection of elastic element mainly happened on the elastic part and the deflection of the contact part is very little. So firstly analyze the deflection of elastic element by assuming that the contact part is rigid body. Then calculate the curve profile of contact part on the elastic element and conduct the simulation via ANSYS software. Finally, compare the desired torque-deflection trajectory with simulation results, and a method of error compensation was proposed to decrease the error of obtained torque-deflection caused by the assumption of rigidity of the contact part.

3.1. Analysis of Deflection of Elastic Element Assuming That the Contact Part Is Rigid Body. In this research, the elastic element is required to deform within a small deflection, so that the mechanical system can be of good elastic performance. Therefore, the deflection of the elastic element can be described using differential equations of the deflection curve. In Figure 2, the elastic element yields the contact force $F\left(F_{x}, F_{y}\right)$ to bear the pressure exerted by the roller. Due to the contact part being viewed as a rigid body, to simplify force analysis, the components of contact forces along $x$-axis and $y$ axis were moved to the extreme of neutral plane of the elastic part. Therefore, the equivalent torque can be generated owing to the forces transformation:

$$
M_{0}=-\left(F_{y} x+y F_{y} \tan \alpha\right) .
$$

According to the differential equations of the deflection curve, the deflection angle and deflection of the extreme of elastic component can be described as the following equations:

$$
\begin{aligned}
& \theta=-\frac{1}{E I}\left[\left(F_{y} x+y F_{y} \tan \alpha\right) l+F_{y} \frac{l^{2}}{2}\right], \\
& v=-\frac{1}{E I}\left[\left(F_{y} x+y F_{y} \tan \alpha\right) \frac{l^{2}}{2}+F_{y} \frac{l^{3}}{3}\right],
\end{aligned}
$$

where $E$ represents Young's modulus of material and $I$ expresses the second moment of area with respect to the $y$ axis, which is determined by the right hand rule.

The geometric relationships shown in Figure 2 can be described as follows:

$$
\begin{aligned}
& x=m_{i} \cos \theta-n_{i} \sin \theta, \\
& y=m_{i} \sin \theta+n_{i} \cos \theta+v .
\end{aligned}
$$

Equations (3) express the coordinates of contact point $\mathrm{B}$ before and after the elastic component deflection. Furthermore, contact point B is not only on the profile of the contact part but also on the profile of the roller. The constraints can 
be obtained by (4). $\gamma$ represents the angle of the parametric equations of the ellipse

$$
\begin{aligned}
& x-x_{c}=a \cos \gamma, \\
& y_{c}-y=b \sin \gamma .
\end{aligned}
$$

The slope of contact point B before the deformation of the elastic element can be expressed by (5). Furthermore, the slope of contact point $B$ after the deformation of the elastic element can be described by taking the derivative of (4), which is shown by (6). Consider

$$
\begin{aligned}
\frac{n_{i-1}-n_{i}}{m_{i-1}-m_{i}} & =\tan (a-\theta), \\
\frac{b}{a} \cot \gamma & =\tan \alpha .
\end{aligned}
$$

The length of the semimajor axis and the semiminor axis of ellipse can be described by (7). At the same time, the coordinates of the center of the roller can be calculated via (8) when the roller moves along the circle around its axis:

$$
\begin{aligned}
a & =r, \\
b & =r \cos \beta, \\
x_{c} & =0, \\
y_{c} & =y_{0}-R \sin \beta .
\end{aligned}
$$

Assume that an external load applied to the system is $\tau$ and reaction forces between the roller and the contact part are generated to equilibrate the system. The relationship between $\tau$ and $F_{y}$ can be obtained via

$$
\tau=F_{y} R
$$

3.2. Design Process of the Nonlinear Stiffness Elastic Element Using MATLAB Software. For a predefined deflection-torque relationship of "low load, low stiffness and high load, high stiffness" which cannot be described using a mathematical function, a numerical method was proposed to solve the adequate curve profile by using MATLAB software. The following part shows the procedure of process for solution of the following available point on the curve profile of the contact part:

Initialization

For $i=1$ : totalNum (total step numbers)

Obtain rotational angel and torque

Resolve equations using $f$ solve function

Get next contact point $\left(m_{i}, n_{i}\right)$ and save it

$m_{i-1}=m_{i}, n_{i-1}=n_{i}$;

End

An interval (iteration step) which affects the accuracy of curve profile was chosen firstly to obtain the number of steps. According to (5), the initial point must be determined. The coordinates of contact point before deformation would be also derived by the rotational angle, torque, and the previous point. When the iteration was over, the resolved points were saved to draw the curve profile.

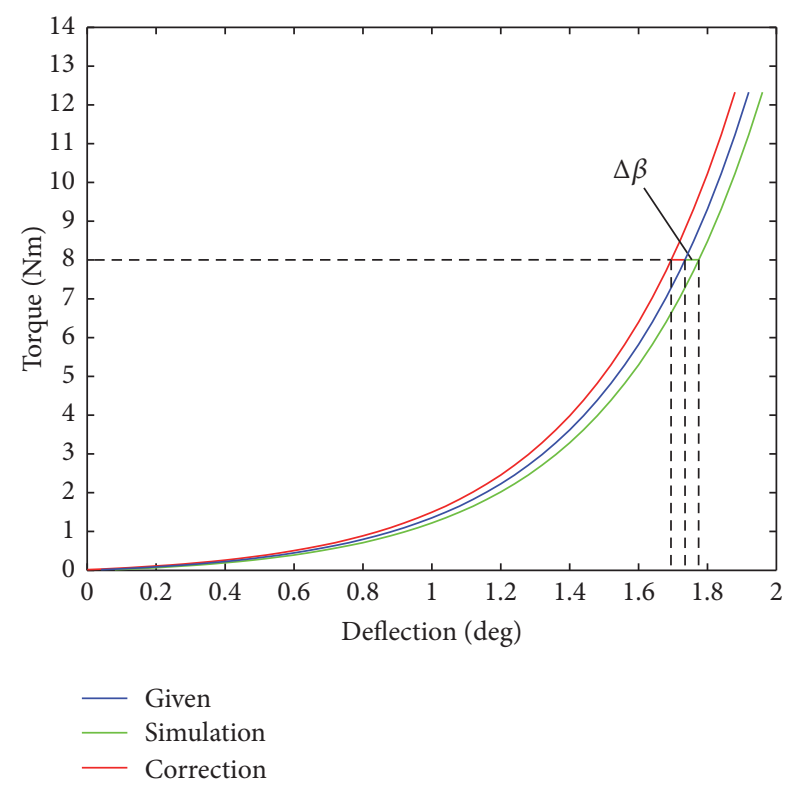

FIGURE 3: Errors analysis of theoretical calculation and simulation results.

3.3. A Method of Error Correction to Compensate the Error of Obtained Torque-Deflection. Since the combination of a cantilever and contact part with a curve profile was used as elastic element of nonlinear elastic system, it is inevitable to refer to the elastic deflection calculation of variable cross section. However, it is difficult to describe this deflection precisely with some algebraic equations.

A simple resolution was proposed to describe the relationship of the deflection and torque in this case. Firstly, analyze the deflection and deflection angle of the cantilever while assuming the contact part is rigid body so that the preliminary shape of the curve profile of the contact part could be derived as mentioned above. According to the simulation results, the derived trajectory of torque-deflection is always a little larger than that derived via theory calculation where the same torque was set. It is not difficult to analyze the reason that the contact part also deflects tinily in horizontal and vertical directions. So some errors should be generated via calculating the geometric equations. Meanwhile, the coordinates of the curve profile of undeformed elastic element were derived via those of deformed elastic element, which makes the derived torque-deflection trajectory of simulation always lower than that derived from the theoretical calculation.

Errors analysis of theoretical calculation and simulation results is shown in Figure 3. The blue curve shows the desired relationship between the torque and deflection and the green curve shows the simulation results. From this figure, it is easy to find that the simulation results are always lower compared to desired trajectory. From different view of this relationship, it would be perfect that the desired trajectory is green curve. So if this curve was set as the desired one, a pseudo desired trajectory should be given firstly such as the desired blue curve for obtaining the green simulated trajectory. Due to the tiny deflection between the desired trajectory and simulation trajectory, there exists linear relationship between the errors 
TABLE 1: The result of optimization.

\begin{tabular}{lc}
\hline Design variable & Optimized value $(\mathrm{mm})$ \\
\hline$l$ & 24.4 \\
$w$ & 3.4 \\
$h$ & 4.9 \\
\hline
\end{tabular}

of pseudo desired trajectory and the errors of desired trajectory. The identical errors $\Delta \beta$ are determined to get the red pseudo desired trajectory. Therefore, the simulation results are likely close to the desired trajectory which is shown as blue curve.

\section{Simulation and Results}

4.1. Optimization Design of the Elastic Part. The stiffness of compliant actuators can be obtained by the curve profile of contact part. However, the inherent stiffness of elastic part has an effect on the range of the stiffness of compliant actuator. It is essential to determine the inherent stiffness firstly. When the inherent stiffness of elastic part is high, the range of the compliant actuator is large. However, the high stiffness generally induces large stress in the elastic element owing to the fact that the inherent stiffness is not fully involved. The inherent stiffness of the elastic part depends on its dimension, and the shape is necessary to be optimized to acquire the appropriate stiffness.

Take initial inherent stiffness of the extreme of the elastic part as optimal object. Its value is higher than the required value which can be calculated via the desired torque-deflection trajectory. The design and optimization of details of contact part were implemented. When the derived shape of the elastic part is available in terms of allowable stress and assembly conditions, the inherent stiffness would be determined. Otherwise, the next inherent stiffness and optimization process would be repeated for the available shape of the elastic part.

Three groups of elastic elements are installed on a plane and each bears the maximum forces $12 \mathrm{Nm}$ within 1.9 degrees in our proposed compliant robotic joint. The dimensions of the cantilever are design variables and the stress is the status variable. The chosen material is $50 \mathrm{CrVA}$ (elasticity modulus $2.06 \mathrm{Gpa}$, poisson ratio: 0.29 , and yield strength: $1.32 \mathrm{Gpa}$ ).

To obtain the optimized structure of the elastic element, the genetic algorithm was used under the constraint conditions of design variables and status variables by using the commercial software ANSYS. The assembly constraint conditions in our proposed structure are $23 \mathrm{~mm}<$ length $<$ $25 \mathrm{~mm}, 3 \mathrm{~mm}<$ width $<3.5 \mathrm{~mm}$ and $3 \mathrm{~mm}<$ height $<5 \mathrm{~mm}$, and stress $<900 \mathrm{Mpa}$. The obtained stiffness of the extreme of the elastic part is $1400 \mathrm{~N} / \mathrm{mm}$. Table 1 shows the details of dimensions.

4.2. The Thickness of the Contact Part. The thickness of the contact part is related to the simulation results of nonlinear stiffness trajectory. Figure 4 shows the thickness of contact part. The thicker the contact part is, the more accurate the

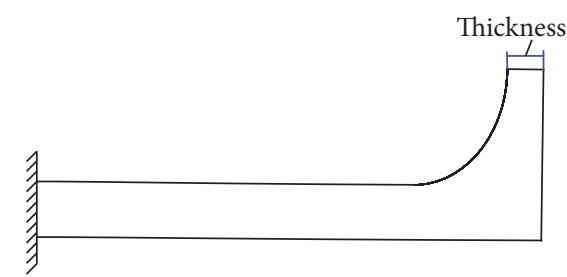

Figure 4: The thickness of the contact part in the elastic element.

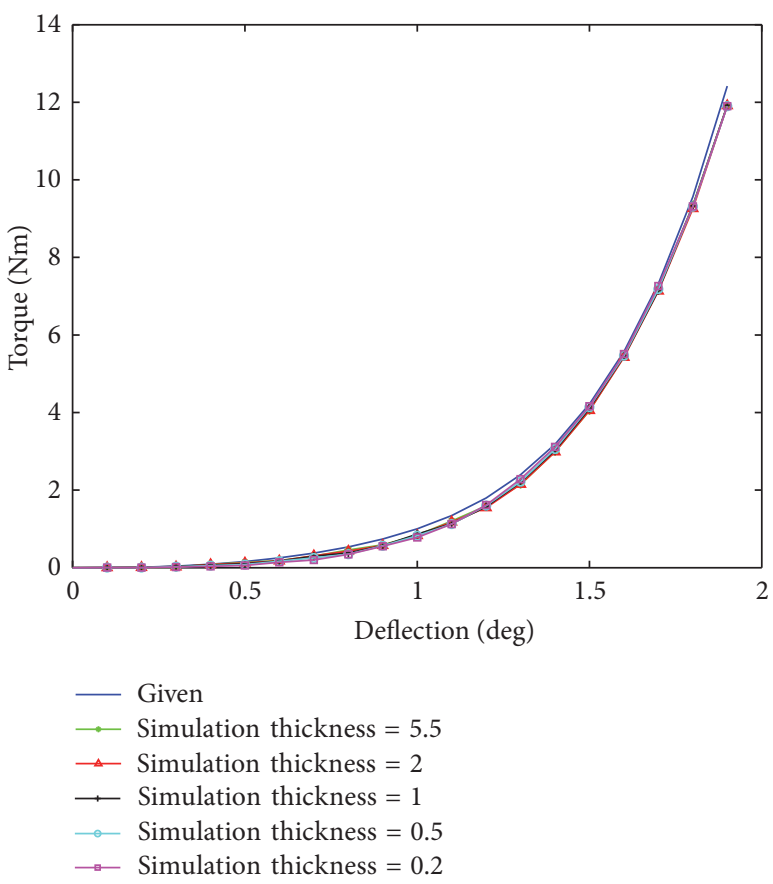

FIGURE 5: The torque-deflection trajectory with different thicknesses.

simulation results of nonlinear stiffness would be, because the deflection of the contact part behaves more approximately to a rigid body. It is also the indicator that whether it is necessary to consider the deflection of contact part during designing such a nonlinear stiffness elastic mechanism. The simulation results are shown in Figure 5, which includes the variation of thickness along $x$-axis from $0.2 \mathrm{~mm}$ to $5.5 \mathrm{~mm}$. From this figure, there is tiny difference for different thicknesses of contact part in the elastic element, which indicates that it is not necessary to consider the influence of the thickness of contact part.

4.3. Error Compensation Using Pseudo Desired Trajectory. For the circumstance of high precision requirement to stiffness characteristic, the error compensation has to be considered, which is induced by the assumption of rigid body of contact part. The method of error compensation is mentioned in Section 3.3, and a pseudo desired trajectory is proposed. Firstly, the uncompensated torque-deflection trajectory is shown in Figure 6. The desired torque-deflection trajectory is exponential format following $\tau=(3 / 20)(10)^{\beta}-3 / 20$, which is shown in blue curve. The pink triangle points are 


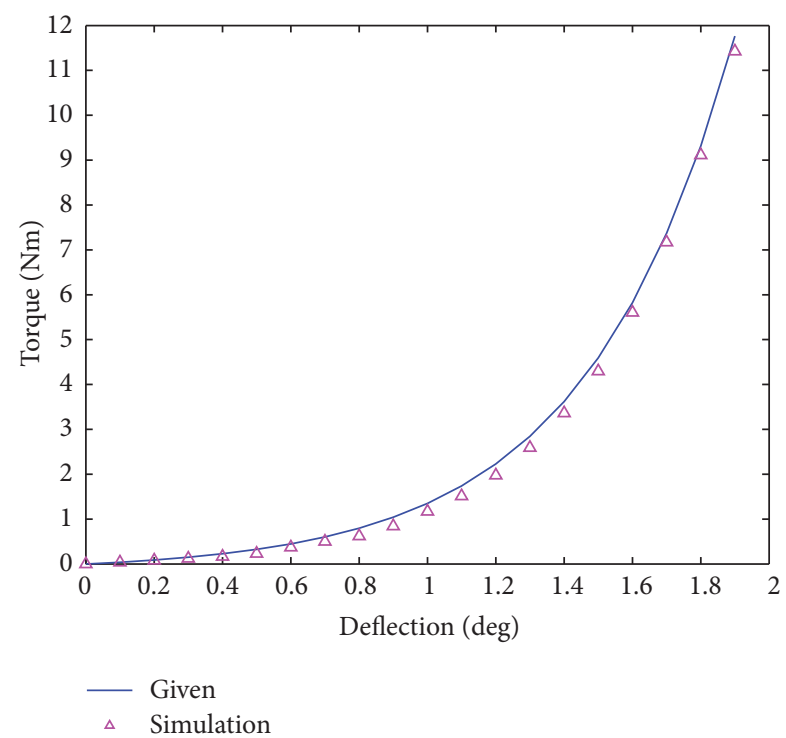

FIGURE 6: Uncompensated torque-deflection trajectory.

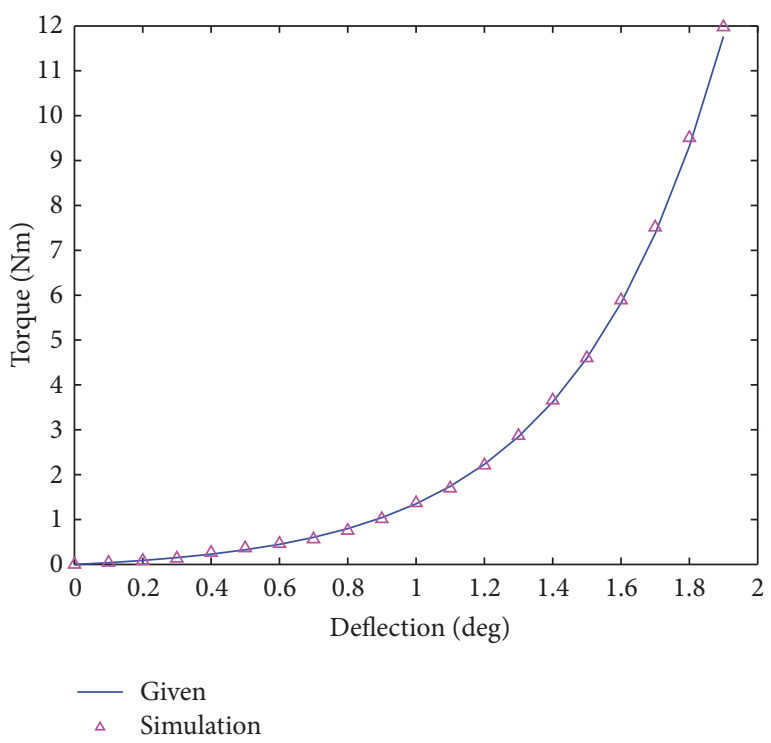

FIGURE 7: Compensated torque-deflection trajectory.

the simulation results derived via ANSYS software. From this figure, the simulation results are regularly lower than the desired trajectory which verifies previous reason analysis of error generation.

The compensated torque-deflection trajectory with the same desired trajectory as Figure 6 is shown in Figure 7, which adapts the pseudo desired trajectory. The simulation results show that compensated torque-deflection trajectory is closer to the desired trajectory than uncompensated one. For comparison between the simulation result using the error compensation method and those not using the error

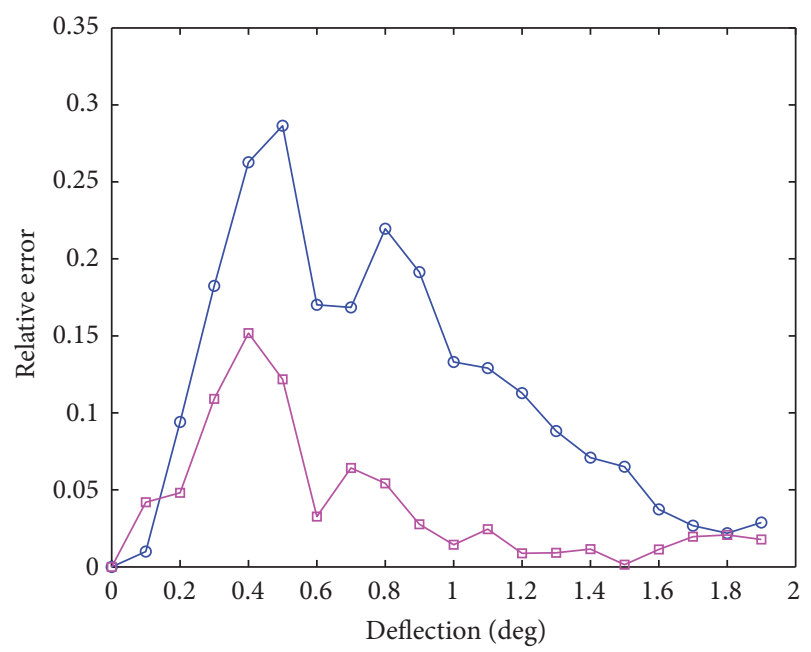

FIGURE 8: Relative errors using and not using compensation method.

compensation method, relative errors are calculated via the following equation:

$$
e_{r}=\frac{\left|\tau_{s}-\tau\right|}{\tau},
$$

where $\tau_{s}$ is the torque derived via simulation and $\tau$ is the theoretical torque.

Figure 8 shows the blue line is the relative errors without compensation between the simulation results and the desired trajectory and the pink line shows the relative errors using the error compensation method. From this figure, the maximum errors of no compensation reach nearly 0.29 . Meanwhile, the errors in the part of small deflection are larger than those in the part of large deflection. With the increasing of torque acting on the elastic element, the relative errors of torque decreased quickly. The relative errors using the error compensation method are lower than those not using the compensation method, and the maximum error derived from the compensation method is 0.15 , only half of that without compensation.

\section{Conclusions}

In this paper, a new design methodology of compliant mechanisms whose stiffness matches the predefined deflectiontorque trajectory of "low load, low stiffness and high load and high stiffness" and an error compensation method were proposed. The assumption that the contact part of elastic element was viewed as rigid body is almost effective in getting the predefined nonlinear stiffness trajectory mentioned above. For obtaining more accurate trajectory, an error compensation method was proposed. According to the simulation results, the proposed method is highly effective in decreasing the relative errors. One joint of the rehabilitation robot for shoulder complex developed by ourselves was illustrated. This research achievement will be useful to design new compliant actuators used in bioinspired robotics, rehabilitation robotics, and so on. 


\section{Abbreviations}

\begin{tabular}{|c|c|}
\hline$\theta$ & The length of cantilever \\
\hline${ }^{\circ}$ & The radius of roller \\
\hline$R:$ & $\begin{array}{l}\text { The radius of circular trajectory of the } \\
\text { roller movement }\end{array}$ \\
\hline a: & $\begin{array}{l}\text { The length of the semimajor axis of ellipse } \\
\text { C }\end{array}$ \\
\hline$b:$ & $\begin{array}{l}\text { The length of the semiminor axis of ellipse } \\
\text { C }\end{array}$ \\
\hline$\omega:$ & The width of cross section of the cantilever \\
\hline h: & $\begin{array}{l}\text { The height of cross section of the } \\
\text { cantilever }\end{array}$ \\
\hline$\gamma$ & $\begin{array}{l}\text { The angle between the slope plane at the } \\
\text { contact point and xaxis }\end{array}$ \\
\hline$\beta:$ & $\begin{array}{l}\text { The rotational angle of roller motion in } \\
\text { terms of the rotatory component where } \\
\text { the elastic element was installed }\end{array}$ \\
\hline$v:$ & Deflection of the end of the elastic part \\
\hline$\theta:$ & $\begin{array}{l}\text { Deflection angle of the end of the elastic } \\
\text { part }\end{array}$ \\
\hline$m_{i-1}, n_{i-1}:$ & $\begin{array}{l}\text { The coordinates of point A before the } \\
\text { deformation of the elastic element }\end{array}$ \\
\hline$m_{i}, n_{i}:$ & $\begin{array}{l}\text { The coordinates of point B before the } \\
\text { deformation of the elastic element }\end{array}$ \\
\hline$x, y:$ & $\begin{array}{l}\text { The coordinates of point B after the } \\
\text { deformation of the elastic element }\end{array}$ \\
\hline$x_{c}, y_{c}:$ & The current coordinates of the roller center \\
\hline & The initial coordinates of the roller center \\
\hline$F_{x}, F_{y}:$ & $\begin{array}{l}\text { The component force of contact forces } \\
\text { between the roller and the elastic element }\end{array}$ \\
\hline & \\
\hline
\end{tabular}

\section{Competing Interests}

The authors declare that they have no competing interests.

\section{Acknowledgments}

The financial support from the Natural Science Foundation of China (Project no. 51475322) and the Programme of Introducing Talents of Discipline to Universities ("111 Program") under Grant no. B16034 and Open Fund of Key Laboratory of Mechanism Theory and Equipment Design of Ministry of Education of China is greatly acknowledged.

\section{References}

[1] G. A. Pratt and M. M. Williamson, "Series elastic actuators," in Proceedings of the IEEE/RSJ International Conference on Intelligent Robots and Systems, vol. 1, pp. 399-406, August 1995.

[2] J. F. Veneman, R. Ekkelenkamp, R. Kruidhof, F. Van der Helm, and H. Van der Kooij, "Design of a series elastic-and bowden cable-based actuation system for use as torque-actuator in exoskeleton-type training," in Proceedings of the IEEE 9th International Conference on Rehabilitation Robotics (ICORR '05), pp. 496-499, 2005.

[3] C. Lagoda, A. C. Schouten, A. H. A. Stienen, E. E. G. Hekman, and H. Van der Kooij, "Design of an electric series elastic actuated joint for robotic gait rehabilitation training," in Proceedings of the 3rd IEEE RAS and EMBS International Conference on Biomedical Robotics and Biomechatronics (BioRob '10), pp. 2126, Tokyo, Japan, September 2010.

[4] G. Carpino, D. Accoto, F. Sergi, N. L. Tagliamonte, and E. Guglielmelli, "A novel compact torsional spring for series elastic actuators for assistive wearable robots," Journal of Mechanical Design, Transactions of the ASME, vol. 134, no. 12, Article ID 121002, 2012.

[5] G. Grioli, S. Wolf, M. Garabini et al., "Variable stiffness actuators: the user's point of view," International Journal of Robotics Research, vol. 34, no. 6, pp. 727-743, 2015.

[6] V. R. Ham, T. G. Sugar, B. Vanderborght, K. W. Hollander, and D. Lefeber, "Compliant actuator designs: review of actuators with passive adjustable compliance/controllable stiffness for robotic applications," IEEE Robotics and Automation Magazine, vol. 16, no. 3, pp. 81-94, 2009.

[7] H. Vallery, J. Veneman, E. van Asseldonk, R. Ekkelenkamp, M. Buss, and H. van Der Kooij, "Compliant actuation of rehabilitation robots," IEEE Robotics \& Automation Magazine, vol. 15, no. 3, pp. 60-69, 2008.

[8] B. Vanderborght, A. Albu-Schaeffer, A. Bicchi et al., "Variable impedance actuators: a review," Robotics and Autonomous Systems, vol. 61, no. 12, pp. 1601-1614, 2013.

[9] A. Jafari, N. G. Tsagarakis, and D. G. Caldwell, "A novel intrinsically energy efficient actuator with adjustable stiffness (AwAS)," IEEE/ASME Transactions on Mechatronics, vol. 18, no. 1, pp. 355-365, 2013.

[10] A. Jafari, N. G. Tsagarakis, and D. G. Caldwell, "AwAS-II: a new actuator with adjustable stiffness based on the novel principle of adaptable pivot point and variable lever ratio," in Proceedings of the IEEE International Conference on Robotics and Automation (ICRA '11), pp. 4638-4643, Shanghai, China, May 2011.

[11] N. G. Tsagarakis, I. Sardellitti, and D. G. Caldwell, "A new variable stiffness actuator (CompAct-VSA): design and modelling," in Proceedings of the 2011 IEEE/RSJ International Conference on Intelligent Robots and Systems: Celebrating 50 Years of Robotics (IROS '11), pp. 378-383, IEEE, San Francisco, Calif, USA, September 2011.

[12] C. Pew and G. K. Klute, "Design of lower limb prosthesis transverse plane adaptor with variable stiffness," Journal of Medical Devices, Transactions of the ASME, vol. 9, no. 3, Article ID 035001, 2015.

[13] S. Wolf, O. Eiberger, and G. Hirzinger, “The DLR FSJ: energy based design of a variable stiffness joint," in Proceedings of the IEEE International Conference on Robotics and Automation (ICRA '11), pp. 5082-5089, May 2011.

[14] W. Friedl, H. Höppner, F. Petit, and G. Hirzinger, "Wrist and forearm rotation of the DLR hand arm system: mechanical design, shape analysis and experimental validation," in Proceedings of the IEEE/RSJ International Conference on Intelligent Robots and Systems (IROS '11), pp. 1836-1842, San Francisco, Calif, USA, September 2011.

[15] R. R. Torrealba and S. B. Udelman, "Design of cam shape for maximum stiffness variability on a novel compliant actuator using differential evolution," Mechanism and Machine Theory, vol. 95, pp. 114-124, 2016.

[16] K. W. Hollander, T. G. Sugar, and D. E. Herring, "Adjustable robotic tendon using a 'jack spring"' in Proceedings of the IEEE 9th International Conference on Rehabilitation Robotics (ICORR '05), pp. 113-118, July 2005.

[17] N. L. Tagliamonte, F. Sergi, D. Accoto, G. Carpino, and E. Guglielmelli, "Double actuation architectures for rendering 
variable impedance in compliant robots: a review," Mechatronics, vol. 22, no. 8, pp. 1187-1203, 2012.

[18] S. A. Migliore, E. A. Brown, and S. P. DeWeerth, "Novel nonlinear elastic actuators for passively controlling robotic joint compliance," Journal of Mechanical Design, vol. 129, no. 4, pp. 406-412, 2007.

[19] G. Palli, G. Berselli, C. Melchiorri, and G. Vassura, "Design of a variable stiffness actuator based on flexures," Journal of Mechanisms and Robotics, vol. 3, no. 3, Article ID 034501, 2011.

[20] S. Rapoport, J. Mizrahi, E. Kimmel, O. Verbitsky, and E. Isakov, "Constant and variable stiffness and damping of the leg joints in human hopping," Journal of Biomechanical Engineering, vol. 125, no. 4, pp. 507-514, 2003.

[21] C. B. Churchill, D. W. Shahan, S. P. Smith, A. C. Keefe, and G. P. McKnight, "Dynamically variable negative stiffness structures," Science Advances, vol. 2, no. 2, Article ID e1500778, 2016.

[22] S. Seok, A. Wang, M. Y. Chuah et al., "Design principles for energy-efficient legged locomotion and implementation on the MIT Cheetah robot," IEEE/ASME Transactions on Mechatronics, vol. 20, no. 3, pp. 1117-1129, 2015.

[23] A. Omer, R. Ghorbani, K. Hashimoto, H.-O. Lim, and A. Takanishi, "A novel design for adjustable stiffness artificial tendon for the ankle joint of a bipedal robot: modeling \& simulation," Machines, vol. 4, no. 1, article 1, 2016.

[24] M. Kilic, Y. Yazicioglu, and D. F. Kurtulus, "Synthesis of a torsional spring mechanism with mechanically adjustable stiffness using wrapping cams," Mechanism and Machine Theory, vol. 57, pp. 27-39, 2012. 


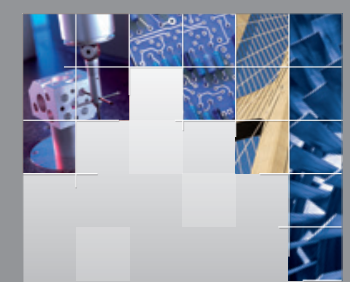

\section{Enfincering}
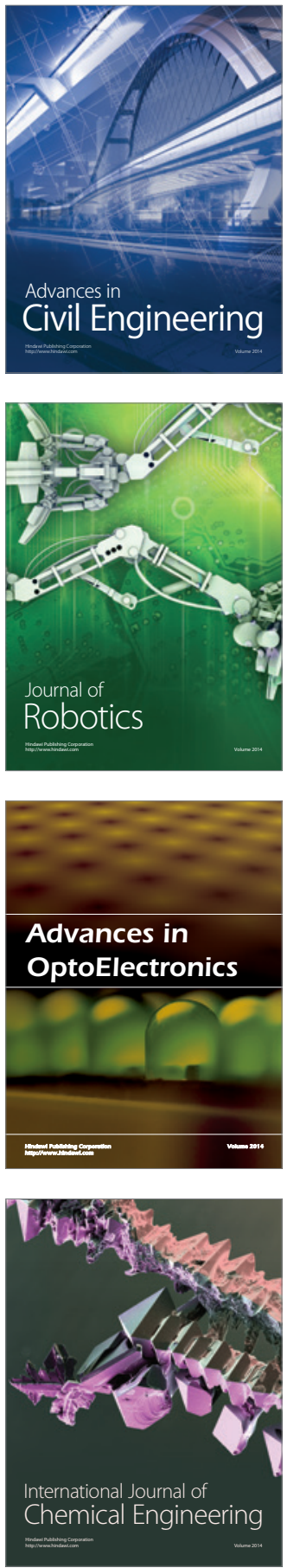

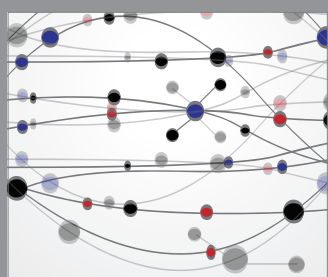

The Scientific World Journal

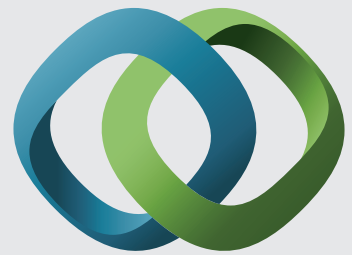

\section{Hindawi}

Submit your manuscripts at

http://www.hindawi.com
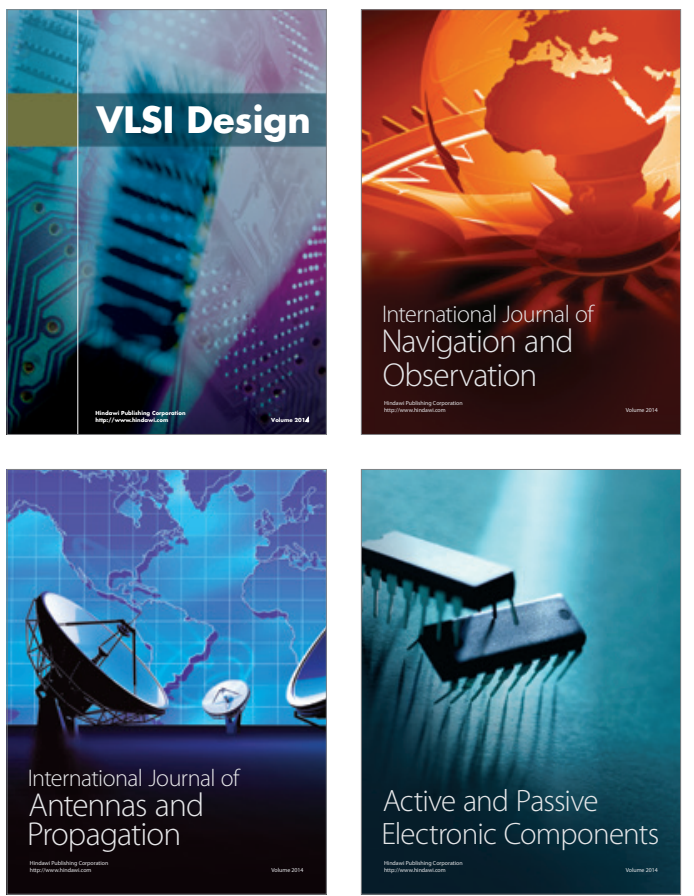
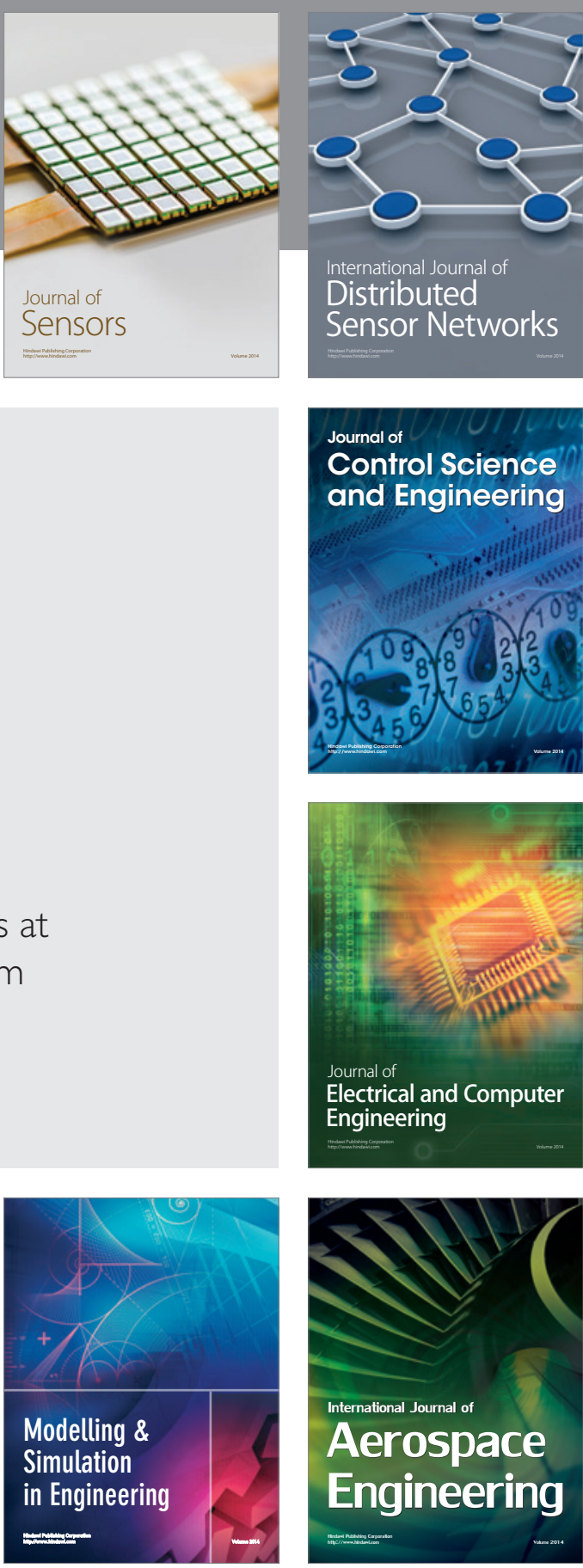

International Journal of

Distributed

Sensor Networks

Journal of

Control Science

and Engineering
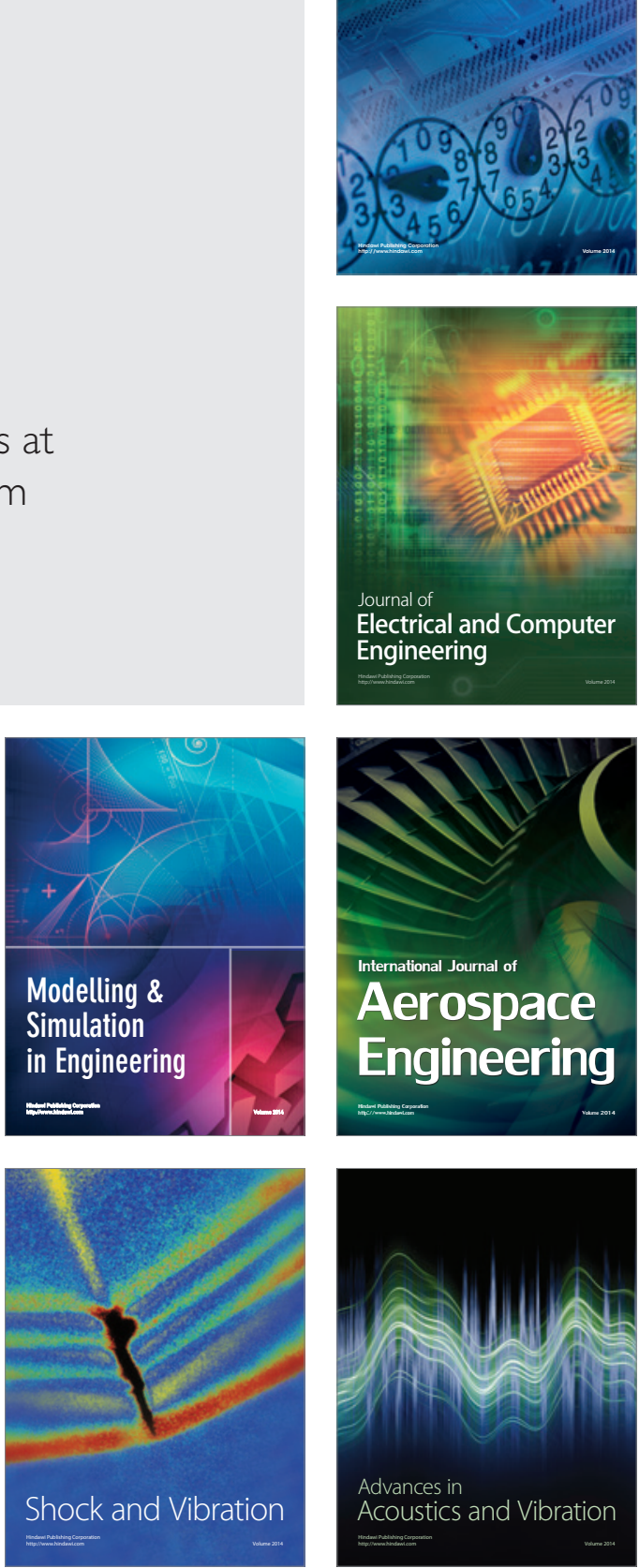